\title{
Recent results from BSM Higgs searches and searches for new light bosons decaying into muon pairs with the CMS detector
}

\author{
Marc Weinberg* \\ CMS Collaboration \\ E-mail: mweinberg2718@gmail.com
}

Several models of new physics predict the existence of new light bosons that are weakly coupled to SM particles. Two examples include SUSY with a "dark" sector (in which the new light bosons can also be relatively long-lived) or models with an extended Higgs sector, e.g. NMSSM. In these scenarios, the new bosons can be produced either in the Higgs decays or as part of SUSY cascades. In the presence of either non-SM Higgs couplings or of additional Higgs bosons, these decays could either hide the additional Higgs bosons from standard searches or alter the measured production rates in standard final states of the SM-like Higgs boson observed at the LHC. Direct searches for non-SM decays of the Higgs boson provide a complementary approach to SM Higgs searches and can help further understand the nature of the Higgs boson by either confirming or rejecting large classes of BSM scenarios. We present the status and recent results of a search for a non-SM Higgs boson decaying to a pair of new light bosons, each of which subsequently decays into a boosted muon pair, using the LHC data collected by the CMS experiment.

XXIII International Workshop on Deep-Inelastic Scattering,

27 April - May 12015

Dallas, Texas

${ }^{*}$ Speaker. 


\section{Recent results from BSM Higgs searches}

The Higgs boson, a direct consequence of the electroweak symmetry breaking mechanism in the standard model (SM), has now been observed at the LHC in multiple decay channels, via several different production mechanisms. The minimal model of the Higgs is very predictive, with the mass $m_{\mathrm{H}}$ the only free parameter. From this it is possible to predict the Higgs production cross section, as well as its partial decay widths. The observed properties of the new particle, including its spin and parity, have thus far been found to be entirely consistent with those expected for the SM Higgs boson.

From an experimental perspective, finding the Higgs boson is only the first step of the Higgs program. The comprehensive measurements of its properties, as well as couplings to the third generation fermions and electroweak bosons is necessary to determine if the observed boson is SM-like in nature. One important aspect of the program is the search for other Higgs boson-like particles, in order to determine if it is part of a spectrum, as predicted by numerous theories such as the next-to-minimal supersymmetric standard model (NMSSM), the 2-Higgs doublet model, and little Higgs models. Another aspect is the use of the Higgs as a portal to new physics. This may be observed through exotic production modes caused by high mass particles decaying to Higgs pairs, or through exotic decay modes like $\mathrm{H} \rightarrow$ invisible or $\mathrm{H} \rightarrow$ aa $\rightarrow 4 \mu$.

The search for the associated production of a single top quark with a Higgs boson, with $\mathrm{H} \rightarrow$ $\mathrm{WW}$, is a very sensitive probe of the sign of the top-Higgs coupling $C_{t}$ [1]. The Higgs is typically irradiated from the heavier legs, either the final state top quark or the $t$-channel $\mathrm{W}$ boson. The strong interference between the two diagrams causes the production cross section to be enhanced for negative couplings. Signal and background contributions are modeled with a multivariate descriminant, and an observed combined limit of $6.7 \times \sigma_{C_{t}=-1}$ is found, as shown in Figure 1 .

It is also becoming feasible to search for the resonant production of pairs of Higgs bosons, as in [2]. Here the diboson pair is assumed to come from a narrow resonance $\mathrm{X}$, and each Higgs is required to decay to a $b \bar{b}$ pair. The $4 \mathrm{~b}$-quark final state is most sensitive to the presence of high mass resonances. The search is performed for both spin- 0 and spin- 2 signal models, with the observed and expected limits in the spin-2 case of Kaluza-Klein (KK) gravitons shown in Figure 2. KK gravitons with masses $380<m_{X}<830 \mathrm{GeV}$ are excluded at a 95\% confidence level.

Another class of searches seeks evidence of extended Higgs sectors, as predicted by models such as the NMSSM. In these models the two complex Higgs doublet states are joined by an additional singlet state, yielding additional bosons that can often be lighter than the known resonance at $125 \mathrm{GeV}$. Ref [3] describes a search for a light Higgs produced in supersymmetric cascade decays that decays subsequently into a $b \bar{b}$ pair. This results in a final state with two $b$ jets, two additional jets, and significant missing transverse energy $E_{\mathrm{T}}^{\text {miss }}$. No significant excess above the SM prediction is observed, and limits are interpreted for a single resonant Higgs-like structure with mass $m_{\mathrm{h}_{1}}$ in Figure 3.

Analogously, searches are carried out at CMS for extended Higgs sectors that result in Higgs bosons heavier than the particle at $125 \mathrm{GeV}$. Heavy Higgs bosons are common in electroweak singlet models, which often predict a high mass scalar particle that participates in symmetry breaking. A very broad search for a heavy Higgs boson in the mass range from 145 to $1000 \mathrm{GeV}$ in the $\mathrm{H} \rightarrow \mathrm{WW}$ and $\mathrm{h} \rightarrow \mathrm{ZZ}$ decay channels is reported in [4]. A large number of fully leptonic and 


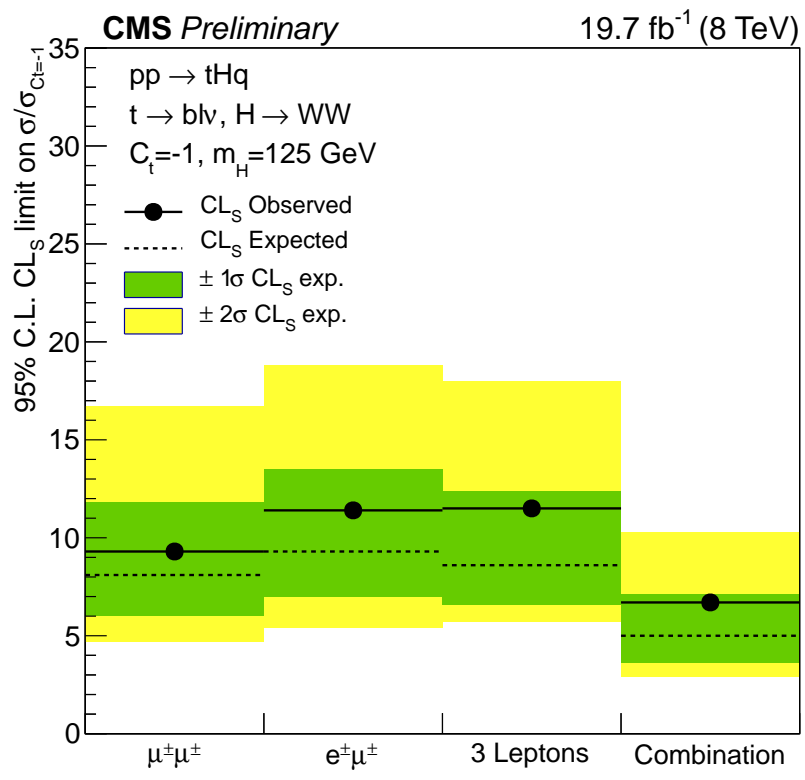

Figure 1: Observed and expected 95\% C.L. upper limit on the Higgs and single top associated production cross section for $C_{t}=-1$. The $\pm 1 \sigma$ (green) and $\pm 2 \sigma$ (yellow) probability bands are also shown.

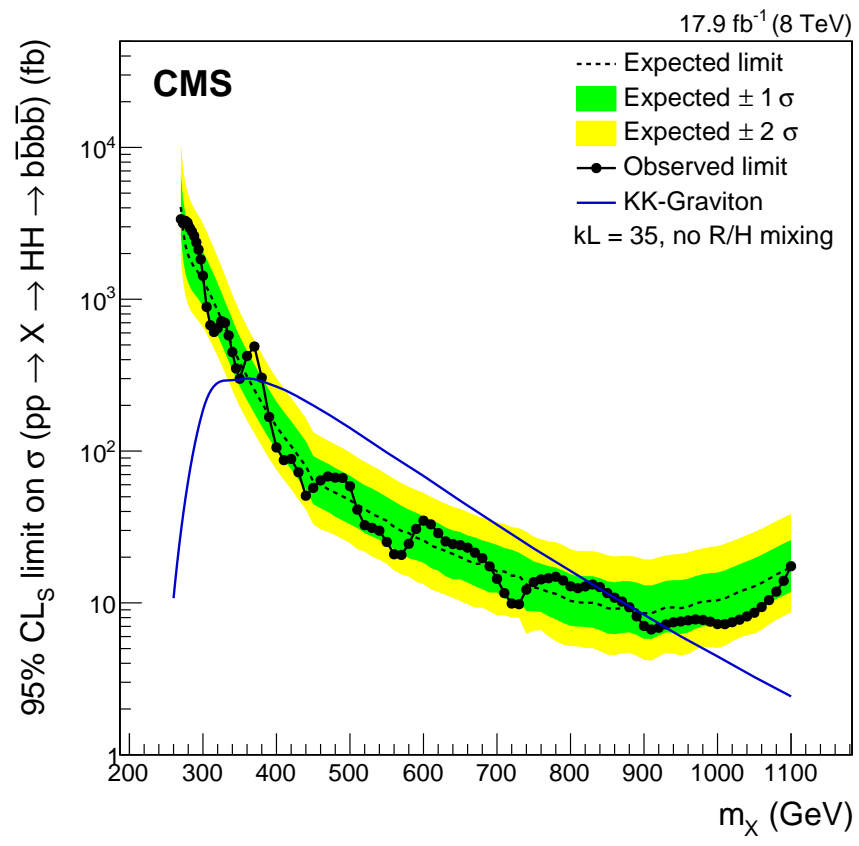

Figure 2: Observed and expected 95\% C.L. upper limit on the cross section for $\mathrm{pp} \rightarrow \mathrm{X} \rightarrow \mathrm{hh} \rightarrow \mathrm{bbbb}$, where the resonance $X$ has spin-2. The theoretical cross section for the first excitation of the KK-graviton for the same parameters is overlaid on the plot. 


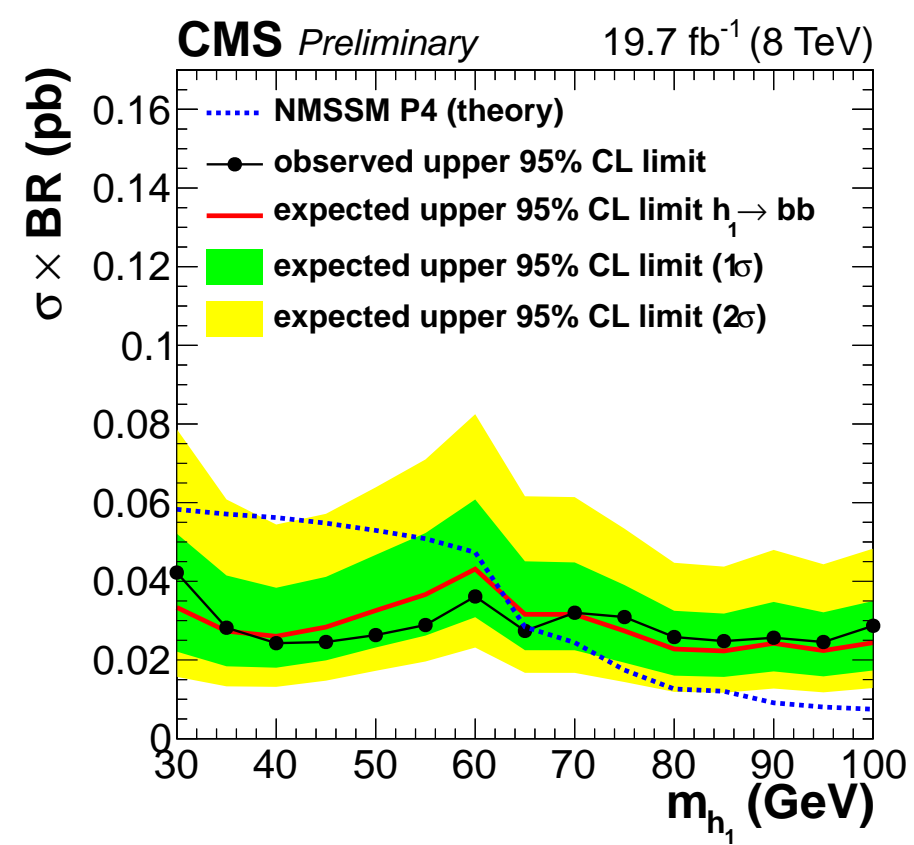

Figure 3: Observed and expected 95\% C.L. upper limit for the light Higgs boson production cross section times branching fraction into b-quarks.

semileptonic decay modes for the diboson pairs are considered, as well as multiple Higgs production mechanisms. The analysis excludes a Higgs boson with SM couplings and decays in the range $145<m_{\mathrm{H}}<1000 \mathrm{GeV}$ at the $95 \%$ confidence level, and the results are interpreted in the context of the heavy Higgs contribution to electroweak symmetry breaking $C^{\prime 2}$ for various contributions to the width of non-SM decays $B_{\text {new }}$, shown in Figure 4.

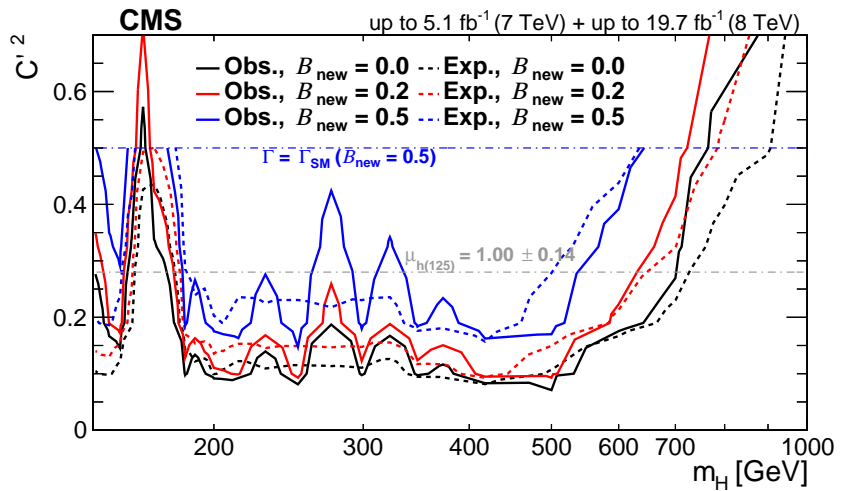

Figure 4: Observed and expected 95\% C.L. upper limits on the electroweak singlet extension of the SM. Upper limits are displayed as a function of the heavy Higgs boson mass and the model parameters $C^{2}$ and $B_{\text {new }}$.

Finally, the BSM Higgs program at CMS includes the search for exotic decays of the Higgs boson. In particular, the invisible decays of a Higgs produced via vector boson fusion [5] provide a sensitive probe of possible new physics that couples to the Higgs sector. Depending on the model, 
this might include decays to neutralinos (SUSY), graviscalars (extra dimensions), or dark matter (via Higgs portal interactions). There is an indirect constraint on undetected decay modes from the measured rates for the visible decay modes, since $\Gamma_{\text {tot }}$ can be measured from the Higgs production rates. Limits are set on the production cross section times invisible branching fraction as a function of the Higgs boson mass in Figure 5. The observed (expected) 95\% C.L. upper limit on the invisible branching fraction at $m_{\mathrm{H}}=125 \mathrm{GeV}$ is found to be $0.57(0.40)$.

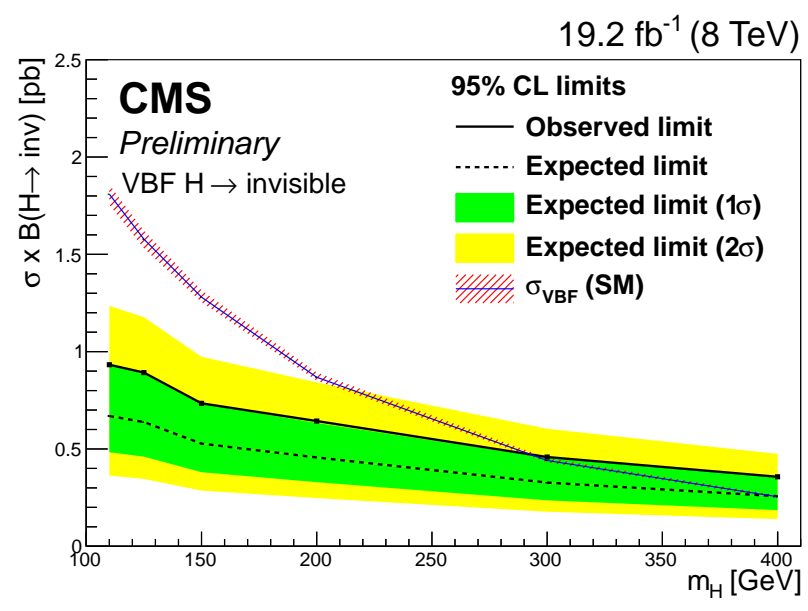

Figure 5: Observed and expected 95\% C.L. upper limit on the cross section times BR(inv) as a function of the Higgs boson mass, assuming SM Higgs boson acceptances.

\section{Muon pairs from new light bosons}

As described above, the presence of unobserved light bosons is a feature of a variety of new physics models. We motivate the search in the $4 \mu$ final state by considering two benchmark scenarios in particular: the NMSSM and dark SUSY.

As discussed in the previous section, the NMSSM is a well motivated minimal extension to the MSSM. It features a more complex Higgs sector consisting of three CP-even states $\left(\mathrm{h}_{1}, \mathrm{~h}_{2}\right.$, and $\mathrm{h}_{3}$ ) and two CP-odd states $\left(\mathrm{a}_{1}\right.$ and $\mathrm{a}_{2}$ ). The inclusion of these additional states, notably the lightest CP-odd state $\mathrm{a}_{1}$, can significantly change the phenomenology of the Higgs sector, as the decay $\mathrm{h}_{1} \rightarrow \mathrm{a}_{1} \mathrm{a}_{1}$ can have a significant branching fraction. We take as our NMSSM benchmarks a set of model points in which each of these CP-odd states decays to a muon pair $\mathrm{a}_{1} \rightarrow 2 \mu$, with mass range $0.25<m_{\mathrm{a}_{1}}<3.55 \mathrm{GeV}$ so that the mass of the $\mathrm{a}_{1}$ lies between twice the mass of the muon and twice the mass of the tau. For these model choices the branching fraction we see characteristic branching fractions to muons of a few percent, with $B R\left(\mathrm{a}_{1} \rightarrow 2 \mu\right) \simeq 7.7 \%$ at $m_{\mathrm{a}_{1}}=2 \mathrm{GeV}$.

Dark SUSY is a new physics scenario with an additional hidden "dark" sector, motivated by observations from satellite experiments like PAMELA and Fermi, which have observed a higher fraction of positrons at high energies. The annihilation of dark matter in the galactic halo has been proposed as a possible explanation for this excess. This typically entails a long-range attractive force acting on slow moving WIMPs. In dark SUSY this is provided by the existence of "dark photons" $\gamma_{D}$, a new boson that couples only weakly to the SM. Our dark SUSY benchmarks consist 
of a set of model points with $0.25<m_{\gamma_{D}}<3.55 \mathrm{GeV}$ and $90<m_{\mathrm{h}_{1}}<150 \mathrm{GeV}$. For these choices we see characteristic branching fractions of a half, with $B R\left(\gamma_{D} \rightarrow 2 \mu\right) \simeq 45 \%$ at $m_{\gamma_{D}}=0.4 \mathrm{GeV}$.

For this analysis [6], events are selected from data taken at CMS by requiring the presence of four muons with $p_{\mathrm{T}}>8 \mathrm{GeV}$ and $|\eta|<2$.4. In addition, to ensure the event is triggered on with high efficiency, at least one muon is required to have $p_{\mathrm{T}}>17 \mathrm{GeV}$ and $|\eta|<0.9$. Each set of oppositely charged muons is then grouped into a dimuon pair if their pairwise invariant mass is greater than $5 \mathrm{GeV}$ and they meet minimal requirements indicating they come from the same vertex. With these choices, individual muons can be shared among multiple dimuon pairs, so we select only events with exactly two dimuon pairs not sharing any common muons. There is no restriction on muons not grouped into pairs ("orphans").

We take $m_{1}$ to be the invariant mass of the dimuon pair with a muon with $p_{\mathrm{T}}>17 \mathrm{GeV}$ and $m_{2}$ to be the remaining dimuon pair. If both pairs contain such a muon, the pairs are randomly assigned to $m_{1}$ and $m_{2}$. The signal region is then defined as a diagonal region in the $m_{1}-m_{2}$ plane, represented by the dashed lines on the right hand side of Figure 6.

The dominant background contributions to the 4 muon final state in the SM come from $b \bar{b}$ events with b-quark decays into $\mu \mu+\mathrm{X}$ via either semileptonic decays or resonances. To estimate this background, the shape of the dimuon invariant mass distribution is measured independently for two dimuon pairs in an orthogonal control region with one dimuon pair and one orphan muon, as shown in Figure 6. A two dimensional template is then constructed from the Cartesian product of each of the one dimensional templates. The other main background for the analysis, from prompt double $\mathrm{J} / \psi$ events, is estimated from normalizing the prediction of the simulation to data.


Figure 6: Left: Comparison of the data failing the $m_{1} \simeq m_{2}$ requirement in the control sample with the prediction of the background shape model scaled to the number of entries in the data. Right: Distribution of the invariant masses $m_{1}$ vs. $m_{2}$ for the isolated dimuon systems for the three events in the data surviving all selections except the $m_{1} \simeq m_{2}$ requirement.

No excess of events is observed with respect to the yields expected from SM processes. A model-independent upper limit of $0.78 \pm 0.05 \mathrm{fb}$ is found for the product of the cross section times 
the branching fraction times acceptance $\sigma(\mathrm{pp} \rightarrow 2 \mathrm{a}+\mathrm{X}) \times B R^{2}(\mathrm{a} \rightarrow 2 \mu) \times \alpha_{\text {gen }}$. Additionally, we determine exclusion limits on Higgs production in both the NMSSM and dark SUSY models as functions of $m_{\mathrm{h}_{1}}$, shown in Figure 7.
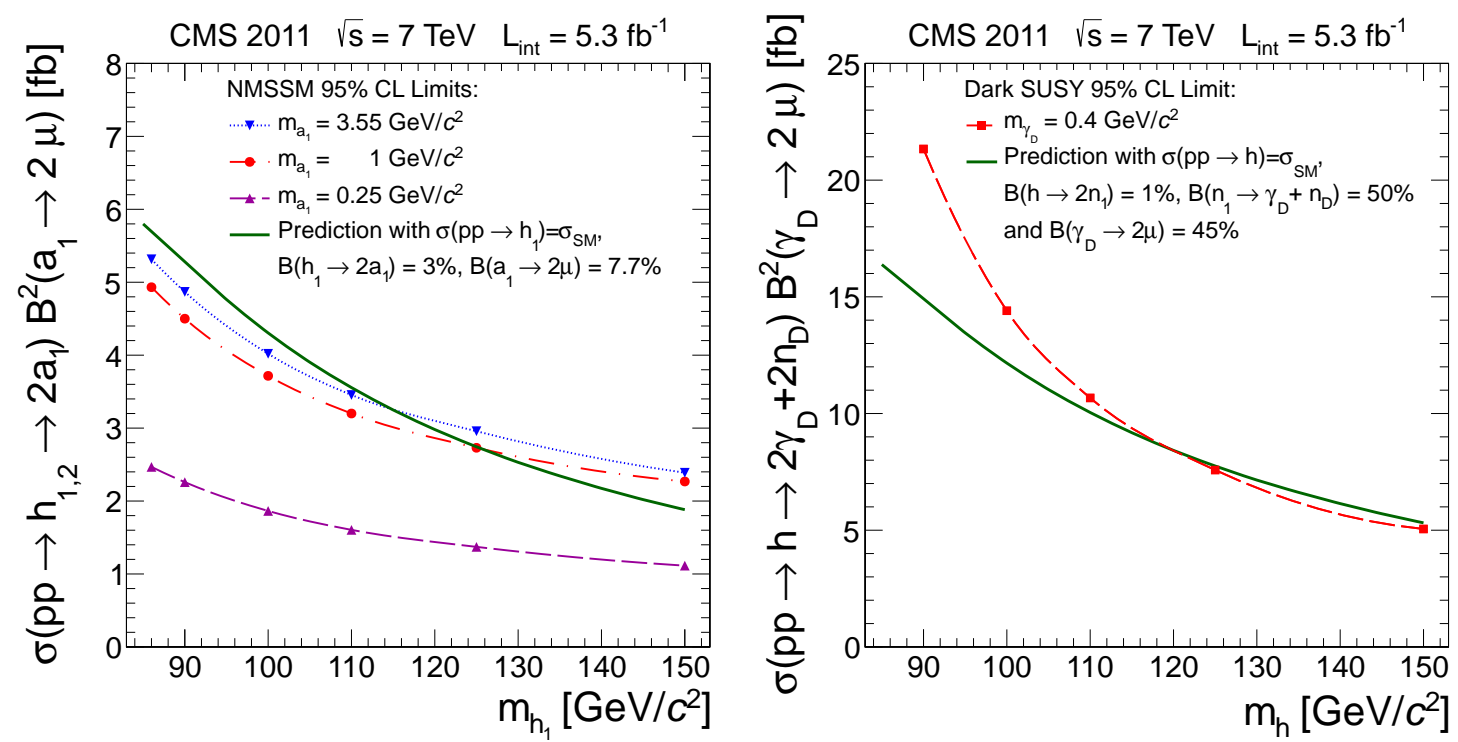

Figure 7: The 95\% C.L. upper limits as functions of $m_{\mathrm{h}_{1}}$ on $\sigma\left(\mathrm{pp} \rightarrow \mathrm{h}_{1} \rightarrow 2 \mathrm{a}_{1}\right) \times \mathrm{BR}^{2}\left(\mathrm{a}_{1} \rightarrow 2 \mu\right)$ for various choices of $m_{\mathrm{a}_{1}}$ in the NMSSM scenario (left) and as a function of $m_{\mathrm{h}}$ on $\sigma\left(\mathrm{pp} \rightarrow \mathrm{h} \rightarrow 2 \gamma_{D}+2 \mathrm{n}_{\mathrm{D}}\right) \times$ $\mathrm{BR}^{2}\left(\gamma_{D} \rightarrow 2 \mu\right)$ with $m_{\gamma_{D}}=0.4 \mathrm{GeV}$ in the dark SUSY scenario (right).

Limits are also found as a function of the new boson mass $m_{\mathrm{a}_{1}}$, as in Figure 8. The purple, red, and blue curves represent different choices of $m_{\mathrm{h}_{1}}$, and the green curve indicates the rate obtained for a toy model with $\sigma\left(\mathrm{pp} \rightarrow \mathrm{h}_{1}\right)=\sigma\left(\mathrm{pp} \rightarrow \mathrm{h}_{2}\right)=\sigma_{\mathrm{SM}}(125 \mathrm{GeV})$.

The CMS experiment supports an extensive program of BSM Higgs searches from Run 1, covering both exotic production and decay modes of the newly discovered Higgs boson. There are several strong limits on new physics from this program, but many decay modes are still largely unconstrained by the $8 \mathrm{TeV}$ data. This, combined with the many well motivated possible extensions to the SM Higgs sector, justifies an optimistic outlook toward Run 2. $13 \mathrm{TeV}$ collisions will significantly improve the reach of the experiment, increasing the precision of measurements of Higgs properties and enhancing the sensitivity of searches for new physics.

\section{References}

[1] S. Chatrchyan et al. [CMS Collaboration], Search for Associated Production of a Single Top Quark and a Higgs Boson in Leptonic Channels, CMS-PAS-HIG-14-026.

[2] S. Chatrchyan et al. [CMS Collaboration], Search for resonant pair production of Higgs bosons decaying to two bottom quark-antiquark pairs in proton-proton collisions at $8 \mathrm{TeV}$, arXiv:1503.04114 [hep-ex].

[3] S. Chatrchyan et al. [CMS Collaboration], Search for a light NMSSM Higgs boson produced in supersymmetric cascades and decaying into a b-quark pair, CMS-PAS-HIG-14-030. 


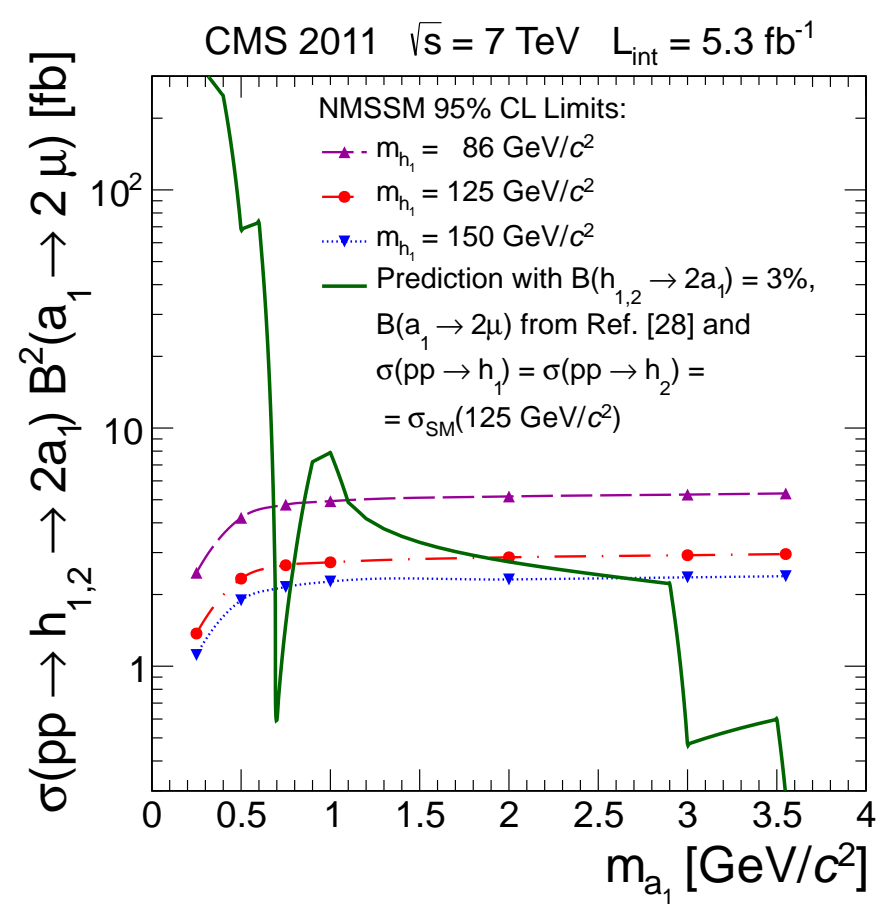

Figure 8: The 95\% C.L. upper limits as functions of $m_{\mathrm{a}_{1}}$ on $\sigma\left(\mathrm{pp} \rightarrow \mathrm{h}_{1} \rightarrow 2 \mathrm{a}_{1}\right) \times \mathrm{BR}^{2}\left(\mathrm{a}_{1} \rightarrow 2 \mu\right)$ for various choices of $m_{\mathrm{h}_{1}}$.

[4] S. Chatrchyan et al. [CMS Collaboration], Search for a Higgs boson in the mass range from 145 to $1000 \mathrm{GeV}$ decaying to a pair of W or Z bosons, arXiv:1504.00936 [hep-ex].

[5] S. Chatrchyan et al. [CMS Collaboration], Search for invisible decays of Higgs bosons in the vector boson fusion production mode, CMS-PAS-HIG-14-038.

[6] S. Chatrchyan et al. [CMS Collaboration], Search for a non-standard-model Higgs boson decaying to a pair of new light bosons in four-muon final states, Phys. Lett. B 726, 564 (2013) [arXiv:1210.7619 [hep-ex]]. 